\title{
Synchronization Theory for Forced Oscillations in Second-Order Systems ${ }^{1}$
}

\author{
J. A. M. BoLLEN ${ }^{2}$
}

Communicated by L. Cesari

\begin{abstract}
We consider differential equations of the form $\ddot{x}+\epsilon f(x, \dot{x})+x=\epsilon u$, where $\epsilon>0$ is supposed to be small. For piecewise continuous controls $u(t)$, satisfying $|u(t)| \leq 1$, we present sufficient conditions for the existence of $2 \pi$-periodic solutions with a given amplitude. We present a method for determining the limiting behavior of controls $\bar{u}_{\epsilon}$ for which the equation has a $2 \pi$-periodic solution with a maximum amplitude and for determining the limit of this maximum amplitude as $\epsilon$ tends to zero. The results are applied to the linear system $\ddot{x}+\epsilon \dot{x}+x=\epsilon u$, the Duffing equation $\ddot{x}+\epsilon\left(\dot{x}+x^{3}\right)+x=\epsilon u$, and the Van der Pol equation $\ddot{x}+\epsilon\left(x^{2}-1\right) \dot{x}+x=\epsilon u$.
\end{abstract}

Key Words. Control theory, synchronization theory, periodic solutions, maximum amplitude.

\section{Introduction}

In this paper, we consider differential equations of the form

$$
\ddot{x}+\epsilon f(x, \dot{x})+x=\epsilon u,
$$

where $\epsilon>0$,

$u \in \Omega:=\{u: \mathbb{R} \rightarrow[-1,+1] \mid u$ is $2 \pi$-periodic and piecewise continuous $\}$, and $f$ is continuously differentiable. We study the behavior of $2 \pi$-periodic solutions $x(t, \epsilon, u)$ of (1) for small $\epsilon>0$ and arbitrary $u \in \Omega$. Especially for small $\epsilon>0$, we ask for a control $\bar{u}_{\epsilon} \in \Omega$ such that (1) admits a $2 \pi$-periodic

\footnotetext{
${ }^{1}$ The author is indebted to M. L. J. Hautus for stimulating discussions and reading the manuscript.

${ }^{2}$ Assistant Professor of Mathematics, Department of Applied Mathematics, Twente University of Technology, Enschede, The Netherlands.
} 
solution $x\left(t, \epsilon, \vec{u}_{\epsilon}\right)$ with a maximum amplitude. Thus, for small $\epsilon>0$, we want to solve the following amplitude maximization problem (AMP):

(AMP) $\max _{u \in \Omega}\left\{\max _{0 \leq t \leq 2 \pi} \mid x(t, \epsilon, u) \| x(t, \epsilon, u)\right.$ is a $2 \pi$-periodic solution of (1)\}.

If $\epsilon>0, u \in \Omega$, and $x(t, \epsilon, u)$ is a corresponding $2 \pi$-periodic solution of (1), then, for any $\tau \in \mathbb{R}$, the shifted control $u(t+\tau)$ is in $\Omega$ and the shifted solution $x(t+\tau, \epsilon, u)$ is a corresponding $2 \pi$-periodic solution of (1). Hence, by shifting the control, it can always be arranged that the maximum amplitude is attained at $t=0$. Therefore, for fixed $\epsilon>0$, we formulate the so-called initial-value maximization problem (IVMP):

(IVMP) $\max _{u \in \Omega}\{|x(0, \epsilon, u)| \mid x(t, \epsilon, u)$ is a $2 \pi$-periodic solution of (1)\}.

If the IVMP has a solution, then the AMP has a solution and vice versa. Moreover, the maximum amplitude equals the maximum (absolute) initial value. It also follows that

$$
\dot{x}\left(0, \epsilon, \bar{u}_{\epsilon}\right)=0,
$$

for a solution where the maximum amplitude is attained at $t=0$. In addition, it follows that a solution of the AMP is not unique. As far as the existence of the maximum amplitude and an optimal control is concerned, we refer to the Appendix and Ref. 1, Chapter 2. The results of this paper are based on a perturbation theorem from the synchronization theory for ordinary differential equations. This theorem is applied to differential equations of the form (1) in Section 2 and is discussed further in Sections 3 and 4, where special attention is paid to the Duffing equation

$$
\ddot{x}+\epsilon\left(\dot{x}+x^{3}\right)+x=\epsilon u \text {. }
$$

In Section 5, we illustrate the results for the linear differential equation

$$
\ddot{x}+\epsilon \dot{x}+x=\epsilon u .
$$

In Section 6, we apply the results to the Van der Pol equation

$$
\ddot{x}+\epsilon\left(x^{2}-1\right) \dot{x}+x=\epsilon u .
$$

\section{Sufficient Conditions for the Existence of $2 \pi$-Periodic Solutions}

In this section, we derive sufficient conditions for the existence of a $2 \pi$-periodic solution of (1) for a given $u \in \Omega$ and small $\epsilon>0$. A well-known perturbation theorem is the following (cf. Ref. 2, Chapter 12). 
Theorem 2.1. Consider the system

$$
\dot{x}=\epsilon f(x, t, \epsilon),
$$

where $f$ is defined and continuously differentiable on $G \times \mathbb{R} \times\left[-\epsilon_{0}, \epsilon_{0}\right]$, with $G$ a region in $\mathbb{R}^{n}$ and $\epsilon_{0}>0$. Let $f$ be periodic in $t$ of period $T$. Let $F: G \rightarrow \mathbb{R}^{n}$ be defined by

$$
F(x):=(1 / T) \int_{0}^{T} f(x, t, 0) d t,
$$

and let $F_{x}$ denote the corresponding functional matrix. If $F(a)=0$ and $F_{x}(a)$ is nonsingular for some fixed $a \in G$, then, for sufficiently small $|\epsilon|$, there exists a unique $T$-periodic solution $x(t, \epsilon)$ of $(2)$, converging to $a$, uniformly with respect to $t$ for $\epsilon \rightarrow 0$.

The proof of this theorem is based on the implicit function theorem. In Ref. 2 , this theorem is also proved for the case where $f$ has finitely many discontinuity points in $t$ on $[0, T]$. The $n$ equations

$$
F(a)=0
$$

are called synchronization equations. In order to be able to apply this theorem to Eq. (1), we describe the solutions by polar coordinates $r$ and $\phi$ in the following way:

$$
x(t)=r(t) \cos (t+\phi(t)), \quad \dot{x}(t)=-r(t) \sin (t+\phi(t)) .
$$

The definition is completed by the conditions

$$
r(0) \geq 0, \quad 0 \leq \phi(0)<2 \pi .
$$

From the differential equation (1), we obtain, in the new coordinates, the system

$$
\begin{aligned}
& \dot{r}=\epsilon[f(r \cos (t+\phi),-r \sin (t+\phi))-u] \sin (t+\phi), \\
& \dot{\phi}=\epsilon r^{-1}[f(r \cos (t+\phi),-r \sin (t+\phi))-u] \cos (t+\phi),
\end{aligned}
$$

while the periodicity conditions

$$
x(0)=x(2 \pi), \quad \dot{x}(0)=\dot{x}(2 \pi)
$$

result in

$$
r(0)=r(2 \pi), \quad \phi(0)=\phi(2 \pi) ;
$$

cf. Subsection 7.4. We have obtained a system of the form (2). In applying Theorem 2.1, we have to exclude $r=0$ in the region $G$, in view of the differential equation for $\phi$. For the function $F: \mathbb{R}^{2} \rightarrow \mathbb{R}^{2}$, depending on $u$, 
we have in this case

$$
F_{u}(r, \phi):=(1 / 2 \pi)\left[\begin{array}{l}
\int_{0}^{2 \pi}[f(r \cos (t+\phi),-r \sin (t+\phi)) \\
-u(t)] \sin (t+\phi) d t \\
r^{-1} \int_{0}^{2 \pi}[f(r \cos (t+\phi),-r \sin (t+\phi)) \\
-u(t)] \cos (t+\phi) d t
\end{array}\right],
$$

where $r$ and $\phi$ have to be considered as real constants. Since the integral over a complete period of a periodic function is invariant with respect to arbitrary shifts of the integrand, the synchronization equations can be written in the form

$$
\alpha(r)=\alpha_{u}(\phi), \quad \beta(r)=\beta_{u}(\phi),
$$

where

$$
\begin{aligned}
& \alpha(r):=\int_{0}^{2 \pi} f(r \cos t,-r \sin t) \sin t d t, \\
& \beta(r):=\int_{0}^{2 \pi} f(r \cos t,-r \sin t) \cos t d t, \\
& \alpha_{u}(\phi):=\int_{0}^{2 \pi} \sin (t+\phi) u(t) d t \\
& \beta_{u}(\phi):=\int_{0}^{2 \pi} \cos (t+\phi) u(t) d t .
\end{aligned}
$$

Now, let $u \in \Omega$, and let $\tilde{r}(\neq 0), \tilde{\phi} \in \mathbb{R}$ satisfy the synchronization equations (6), and let also

$$
\operatorname{det} F_{u, x}(\tilde{r}, \tilde{\phi}) \neq 0 \text {. }
$$

Then, according to Theorem 2.1 and the transformation (3), it follows that, for sufficiently small $\epsilon>0$, the differential equation (1) with control $u$ admits $2 \pi$-periodic solutions $x(t, \epsilon, u)$, converging to $\tilde{r} \cos (t+\tilde{\phi})$, for $\epsilon \downarrow 0$ uniformly with respect to $t$. A point $(\tilde{r}, \tilde{\phi})$ satisfying the previous conditions is called a synchronization point of $u$.

\section{Synchronization Points}

In this section, we show how to determine synchronization points for a given $u \in \Omega$. The Duffing equation

$$
\ddot{x}+\epsilon\left(\dot{x}+x^{3}\right)+x=\epsilon u
$$


is used as a concrete example. From the definition of $\alpha(r)$, it follows that

$$
\begin{aligned}
\alpha(r) & =\int_{0}^{2 \pi} f(r \cos (t+\pi),-r \sin (t+\pi)) \sin (t+\pi) d t \\
& =-\int_{0}^{2 \pi} f(-r \cos t,+r \sin t) \sin t d t=-\alpha(-r) .
\end{aligned}
$$

Similarly,

$$
\beta(r)=-\beta(-r)
$$

Hence, the function

$$
\operatorname{sc}(r):=(\alpha(r), \beta(r))
$$

is odd,

$$
(\operatorname{sc}(r)=-\operatorname{sc}(-r)) .
$$

From the definition of $\alpha_{u}(\phi)$ and $\beta_{u}(\phi)$, it follows that

$$
\left[\begin{array}{l}
\alpha_{u}(\phi) \\
\beta_{u}(\phi)
\end{array}\right]=\left[\begin{array}{cc}
\cos \phi & \sin \phi \\
-\sin \phi & \cos \phi
\end{array}\right]\left[\begin{array}{l}
\alpha_{u} \\
\beta_{u}
\end{array}\right]
$$

where

$$
\begin{aligned}
& \alpha_{u}:=\alpha_{u}(0)=\int_{0}^{2 \pi} \sin t u(t) d t \\
& \beta_{u}:=\beta_{u}(0)=\int_{0}^{2 \pi} \cos t u(t) d t .
\end{aligned}
$$

Thus, the point $\left(\alpha_{u}(\phi), \beta_{u}(\phi)\right)$ is obtained by rotating $\left(\alpha_{u}, \beta_{u}\right)$ clockwise over an angle $\phi$ around the origin. In order to identify the set $\left\{\left(\alpha_{u}, \beta_{u}\right) \mid u \in \Omega\right\}$, we first define a subclass of controls $u \in \Omega$. Let $s \in[0,2 \pi)$. Then, the control $\mu_{s} \in \Omega$ is defined by

$$
u_{s}(t):=\operatorname{sign}(\sin (t-s)) .
$$

Such a control is called bang-banger. More generally, a control $u \in \Omega$ for which

$$
u(t)=+1 \quad \text { or } \quad u(t)=-1,
$$

for every $t \in \mathbb{R}$, is called a bang-bang control. A discontinuity point of a bang-bang control is called a switch point. Hence, a bang-banger is a bang-bang control for which the distance between two consecutive switch points equals $\pi$. 
Theorem 3.1. $\left\{\left(\alpha_{u}, \beta_{u}\right) \mid u \in \Omega\right\}=\left\{(x, y) \in \mathbb{R}^{2} \mid x^{2}+y^{2} \leq 16\right\}$.

Proof. Each point $(x, y)$ satisfying

$$
x^{2}+y^{2} \leq 16
$$

can be written in the form $(\rho \cos s,-\rho \sin s)$, where

$$
\rho \in[0,4], \quad s \in[0,2 \pi) .
$$

Define

$$
\bar{u}:=(1 / 4) \rho \mu_{s} .
$$

Then, $\bar{u} \in \Omega$, and it is easy to verify that

$$
\left(\alpha_{\bar{u}}, \beta_{\bar{u}}\right)=(\rho \cos s,-\rho \sin s) .
$$

On the other hand, let $u \in \Omega$, and let $\rho \geq 0$ and $s \in[0,2 \pi)$ be defined by the relation

$$
\rho e^{i s}=\beta_{u}+i \alpha_{u}
$$

Since $\rho \in \mathbb{R}$, we have

$$
\begin{aligned}
\rho & =e^{-i s}\left(\beta_{u}+i \alpha_{u}\right)=e^{-i s} \int_{0}^{2 \pi}(\cos t+i \sin t) u(t) d t \\
& =\int_{0}^{2 \pi} e^{i(t-s)} u(t) d t=\int_{0}^{2 \pi} \cos (t-s) u(t) d t .
\end{aligned}
$$

Therefore,

$$
\rho \leq \int_{0}^{2 \pi}|\cos (t-s)| d t=4 ;
$$

thus,

$$
\alpha_{u}^{2}+\beta_{u}^{2} \leq 16 .
$$

From the first part of the proof, it even follows that

$$
\left\{\left(\alpha_{u}, \beta_{u}\right) \mid u \in \Omega\right\}=\left\{\left(\alpha_{u}, \beta_{u}\right) \mid u=\rho \mu_{s}, \rho \in[0,1], s \in[0,2 \pi)\right\} .
$$

The part of the circle

$$
x^{2}+y^{2}=16
$$

in the upper half plane is reached by bang-bangers $\mu_{s}, \pi<s<2 \pi$, whereas the part of the circle

$$
x^{2}+y^{2}=16
$$


in the lower half plane is reached by bang-bangers $\mu_{s}, 0<s<\pi$. The points $(4,0)$ and $(-4,0)$ are reached by $\mu_{0}$ and $\mu_{\pi}$, respectively. Now that we know $\left\{\left(\alpha_{u}, \beta_{u}\right) \mid u \in \Omega\right\}$, it is easy to find a point $(\tilde{r}, \tilde{\phi})$ satisfying the synchronization equations (6) for a given $u \in \Omega$. We deal with a concrete example, viz., the Duffing equation

$$
\ddot{x}+\epsilon\left(\dot{x}+x^{3}\right)+x=\epsilon u \text {. }
$$

For this case,

$$
f(x, \dot{x})=\dot{x}+x^{3}
$$

hence, by definition,

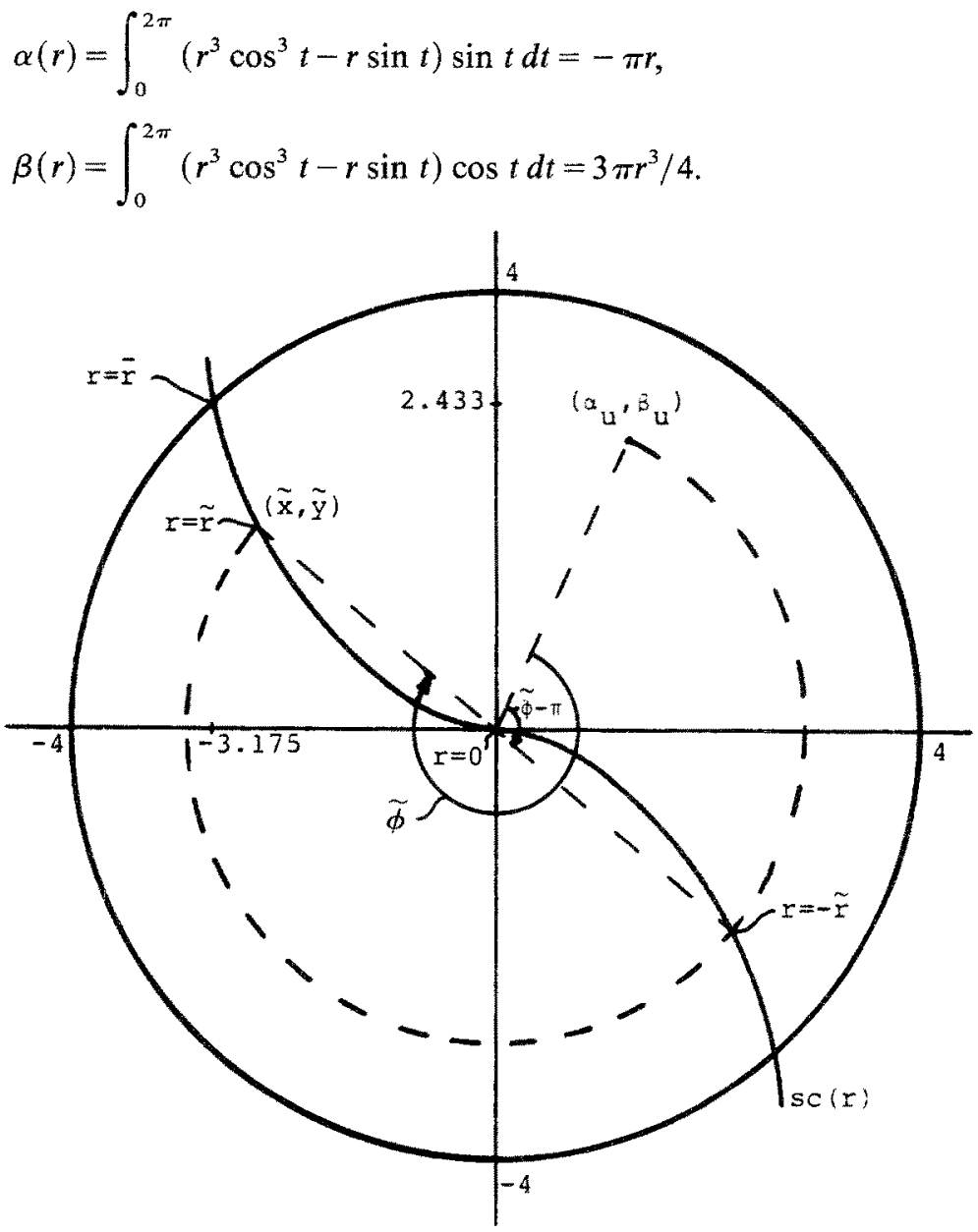

Fig. 1. Synchronization curve for the Duffing equation. 
Consequently, the so-called synchronization curve

$$
\operatorname{sc}(r)=(\alpha(r), \beta(r))
$$

satisfies the equation

$$
4 \pi^{2} y=-3 x^{3}
$$

see Fig. 1.

Now, let $u \in \Omega$, and calculate $\alpha_{u}$ and $\beta_{u}$ from (9). The circle with center 0 and passing through $\left(\alpha_{u}, \beta_{u}\right)$ (the dotted circle part) intersects $\operatorname{sc}(r)$ in the point $(\tilde{x}, \tilde{y})$, with $\tilde{x}<0$. Determine the angle $\tilde{\phi}$ as indicated in Fig. 1. Determine $\tilde{r}>0$, satisfying

$$
\operatorname{sc}(r)=(\tilde{x}, \tilde{y}) .
$$

Now, by virtue of $(8)$, we have

$$
\left(\alpha_{u}(\tilde{\phi}), \beta_{u}(\tilde{\phi})\right)=(\tilde{x}, \tilde{y})=(\alpha(\tilde{r}), \beta(\tilde{r})) ;
$$

hence, $(\tilde{r}, \tilde{\phi})$ satisfies the synchronization equations. Moreover, if $\operatorname{det} F_{u, x}(\tilde{r}, \tilde{\phi}) \neq 0$,

then $(\tilde{r}, \tilde{\phi})$ is a synchronization point of $u$.

Remark 3.1. Note that

$$
\left(\alpha_{u}(\tilde{\phi}-\pi), \beta_{u}(\tilde{\phi}-\pi)\right)=-(\tilde{x}, \tilde{y})=(\alpha(-\tilde{r}), \beta(-\tilde{r})) ;
$$

therefore, $(-\tilde{r}, \tilde{\phi}-\pi)$ also satisfies the synchronization equations. This is not surprising, since, if $r(t), \phi(t)$ is a $2 \pi$-periodic solution of the system (4), then $-r(t), \phi(t)-\pi$ is also a $2 \pi$-periodic solution of the system (4). The two corresponding $2 \pi$-periodic solutions of (1) coincide; hence, we only concentrate on $(\tilde{r}, \tilde{\phi})$. Furthermore, for the shifted control

$$
\bar{u}(t):=u(t+\tau), \quad \tau \in \mathbb{R},
$$

we have

$$
\begin{aligned}
\alpha_{\tilde{u}}(\tilde{\phi}+\tau) & =\int_{0}^{2 \pi} \sin (t+\tilde{\phi}+\tau) u(t+\tau) d t=\int_{0}^{2 \pi} \sin (t+\tilde{\phi}) u(t) d t \\
& =\alpha_{u}(\tilde{\phi}) .
\end{aligned}
$$

Similarly,

$$
\beta_{\bar{u}}(\tilde{\phi}+\tau)=\beta_{u}(\tilde{\phi}) .
$$

Consequently, $(\tilde{r}, \tilde{\phi}+\tau)$ satisfies the synchronization equations for $u$. This agrees with the fact that, if $r(t), \phi(t)$ is a $2 \pi$-periodic solution of the system 
(4) for the control $u$, then $r(t+\tau), \phi(t+\tau)+\tau$ is a $2 \pi$-periodic solution of the system (4) for the control $\bar{u}$, which, in turn, agrees with the shifting property of controls $u$ and periodic solutions $x$, mentioned in Section 1 for the differential equation (1).

We now consider det $F_{u, x}(r, \phi)$. From (5) and the definitions in (7), it follows that, for arbitrary $r, \phi \in \mathbb{R}$, we have

$$
\begin{aligned}
\left(4 \pi^{2} r\right) \operatorname{det} F_{u, x}(r, \phi) & =\alpha_{u}^{\prime}(\phi) \beta^{\prime}(r)-r^{-1} \alpha_{u}^{\prime}(\phi)\left(\beta(r)-\beta_{u}(\phi)\right) \\
& -\beta_{u}^{\prime}(\phi) \alpha^{\prime}(r),
\end{aligned}
$$

where the prime means differentiation with respect to the argument. Since

$$
\alpha_{u}^{\prime}(\phi)=\beta_{u}(\phi), \quad \beta_{u}^{\prime}(\phi)=-\alpha_{u}(\phi),
$$

it follows that, for a point $(\tilde{r}, \tilde{\phi})$ satisfying the synchronization equations, we have

$$
\left(4 \pi^{2} \tilde{r}\right) \operatorname{det} F_{u, x}(\tilde{r}, \tilde{\phi})=\alpha^{\prime}(\tilde{r}) \alpha(\tilde{r})+\beta^{\prime}(\tilde{r}) \beta(\tilde{r}) .
$$

Hence, for a point $(\tilde{r}, \tilde{\phi})$ satisfying the synchronization equations,

$$
\operatorname{det} F_{u, x}(\tilde{r}, \tilde{\phi})=0, \quad \text { iff }\left(\alpha^{2}(r)+\beta^{2}(r)\right)^{\prime}=0 \text {, at } r=\tilde{r} \text {, }
$$

or equivalently iff the square of the Euclidean length of the vector $\operatorname{sc}(r)$ is stationary at $r=\tilde{r}$. Usually, in such a point the synchronization curve will be orthogonal to the radius. For the Duffing equation, we have

$$
\alpha^{2}(r)+\beta^{2}(r)=\pi^{2}\left(r^{2}+(9 / 16) r^{6}\right) ;
$$

therefore,

$$
\operatorname{det} F_{u, x}(\tilde{r}, \tilde{\phi})=\left(16+27 \tilde{r}^{4}\right) / 64>0 .
$$

Consequently, every point $(\tilde{r}, \tilde{\phi}), \tilde{r} \neq 0$, satisfying the synchronization equations for some $u \in \Omega$ is a synchronization point of $u$.

Remark 3.2. Since, for arbitrary $r, \phi \in \mathbb{R}$,

$$
\begin{aligned}
\left(\alpha_{-u}(\phi), \beta_{-u}(\phi)\right) & =-\left(\alpha_{u}(\phi), \beta_{u}(\phi)\right)=-(\alpha(r), \beta(r)) \\
& =(\alpha(-r), \beta(-r)),
\end{aligned}
$$

it follows that $(-\tilde{r}, \tilde{\phi})$ is a synchronization point of the control $-u$. If the function $f$ is $o d d$,

$$
f(x, y)=-f(-x,-y) \text {; }
$$

this is not surprising; in that case, if $r(t), \phi(t)$ is a $2 \pi$-periodic solution of the system (4) for the control $u$, then $-r(t), \phi(t)$ is a $2 \pi$-periodic solution of the system (4) for the control $-u$. However, if $f$ is not odd, then this is 
not necessarily true (cf. Section 5). If the function $f$ is even,

$$
f(x, y)=f(-x,-y),
$$

then

$$
\begin{aligned}
\alpha(-r) & =\int_{0}^{2 \pi} f(-r \cos t, r \sin t) \sin t d t \\
& =\int_{0}^{2 \pi} f(r \cos t,-r \sin t) \sin t d t=\alpha(r) ;
\end{aligned}
$$

and, since

$$
\alpha(-r)=-\alpha(r),
$$

even for arbitrary $f$, it follows that

$$
\alpha(r) \equiv 0 .
$$

Similarly,

$$
\beta(r)=0 .
$$

Consequently,

$$
\operatorname{det} F_{u, x}(r, \phi)=0 \text {, }
$$

for every point $(r, \phi)$ satisfying the synchronization equations; hence, Theorem 2.1 gives no information if $f$ is even. If the function

$$
f(x, y)=\frac{1}{2}[f(x, y)-f(-x,-y)]+\frac{1}{2}[f(x, y)+f(-x,-y)]
$$

contains a nonzero odd and a nonzero even part, then $\alpha(r)$ and $\beta(r)$ do depend only on the odd part, and so do the synchronization points $(\tilde{r}, \tilde{\phi})$. Consequently, for fixed $u \in \Omega$, the limiting $2 \pi$-periodic solution $\tilde{r} \cos (t+\tilde{\phi})$ of (1) does not depend on the even part of $f$. However, the $2 \pi$-periodic solutions of (1) itself will depend in general, for fixed $u \in \Omega$ and $\epsilon>0$, on the odd and even parts of $f$. This will be shown in Section 5 for the linear system

$$
\ddot{x}+\epsilon(a+\dot{x})+x=\epsilon u, \quad a \in \mathbb{R} .
$$

\section{Maximum Amplitude and Optimal Controls}

So far, we have considered arbitrary $u \in \Omega$ and pointed out how to find values $\tilde{r}, \tilde{\phi}$ such that, for sufficiently small $\epsilon>0$, Eq. (1) with the control $u$ has $2 \pi$-periodic solutions $x(t, \epsilon, u)$ uniformly converging to $\tilde{r} \cos (t+\tilde{\phi})$ 
for $\epsilon \downarrow 0$. For small $\epsilon>0$, the value of $|\tilde{r}|$ is an approximation of the amplitude of these $2 \pi$-periodic solutions. Since we are searching for $2 \pi$-periodic solutions with maximum amplitude, it seems to make sense to determine controls $u$ in $\Omega$ which yield synchronization points $(\tilde{r}, \tilde{\phi})$ with maximum $|\tilde{r}|$. The maximum $|\tilde{r}|$ for a synchronization point $(\tilde{r}, \tilde{\phi})$ equals the maximum $|\hat{r}|$ for which

$$
\operatorname{sc}(\hat{r}) \in\left\{(x, y) \in \mathbb{R}^{2} \mid x^{2}+y^{2}=16\right\},
$$

at least if

$$
\text { det } F_{u, x}(\hat{r}, 0) \neq 0 \text {. }
$$

To avoid the need of distinguishing between positive and negative $\tilde{r}$ and to avoid the complications resulting from it, we assume from now on that $f$ is an odd function. Then, according to the related part of Remark 3.3, we can restrict ourselves to the maximization of $\tilde{r}$. One may wonder to what extent the maximum $\tilde{r}$ is an approximation of the maximum amplitude for small $\epsilon>0$, since the synchronization theory constitutes only a sufficient condition for the existence of $2 \pi$-periodic solutions for small $\epsilon>0$. In the next theorem, we present conditions under which the maximum $\tilde{r}$ is the limiting value of the maximum amplitude as $\epsilon \downarrow 0$. For a complete proof, we refer to the Appendix.

Theorem 4.1. Let the function $f$ be odd and twice continuously differentiable, and let the initial values $x_{0}, \dot{x}_{0}$ of $2 \pi$-periodic solutions of the differential equation (1) be uniformly bounded for all $2 \pi$-periodic, measurable controls $u,|u(t)| \leq 1$, and small $\epsilon>0$. Furthermore, let

$$
\bar{r}:=\max \left\{r \in \mathbb{R} \mid \alpha^{2}(r)+\beta^{2}(r)=16\right\}
$$

exist, and assume that, for $r=\bar{r}$, there holds

$$
(d / d r)\left(\alpha^{2}(r)+\beta^{2}(r)\right) \neq 0 .
$$

Then, for the differential equation (1), we have:

(i) the maximum amplitude $A_{\epsilon}$ exists for each sufficiently small $\epsilon>0$ and $A_{\epsilon} \rightarrow \bar{r}(\epsilon \downarrow 0)$;

(ii) for sufficiently small $\epsilon>0$, the optimal controls $\bar{u}_{\epsilon} \in \Omega$ are bangbang controls with two switch points on $[0,2 \pi)$;

(iii) there is exactly one bang-banger $\bar{u} \in \Omega$, such that

$$
\alpha_{\bar{u}}=\alpha(\bar{r}), \quad \beta_{\bar{u}}=\beta(\bar{r}) ;
$$

(iv) the optimal controls $\bar{u}_{\epsilon} \in \Omega$ for which there exists a $2 \pi$-periodic 
solution with the maximum amplitude attained at $t=0$ satisfy

$\int_{0}^{2 \pi}\left|\bar{u}_{\epsilon}(t)-\bar{u}(t)\right| d t \rightarrow 0, \quad \epsilon \downarrow 0$.

We first give some comments. The uniform boundedness condition in this theorem is a rather obvious necessary condition, with the exclusion of the fact that it must hold not only for all piecewise continuous controls, but for all measurable controls. The measurability concept often appears in optimal control problems for proving closedness of reachable sets (see e.g., Ref. 3), and so it did in the proof of Theorem 4.1 in order to prove (i).

The result (ii) follows from a result on the minimal distance of successive zeros of differential equations of the type

$$
\ddot{x}+w \dot{x}+(1+v) x=0,
$$

where $w(t)$ and $v(t)$ are small continuous functions.

If $\bar{r}$, as defined in (11), exists, then obviously sc $(r)$ is a boundary point of $\left\{(x, y) \in \mathbb{R}^{2} \mid x^{2}+y^{2} \leq 16\right\}$, and then (iii) follows directly from the proof of Theorem 3.1. From (12) and (13), it follows that $(\bar{r}, 0)$ is a synchronization point of $\bar{u}$.

For the $2 \pi$-periodic solutions $x\left(t, \epsilon, \bar{u}_{\varepsilon}\right)$ of (iv), there holds

$$
\dot{x}\left(0, \epsilon, \bar{u}_{\epsilon}\right)=0 ;
$$

(cf. Section 1). According to (ii), the controls $\bar{u}_{\epsilon}$ of (iv) have two switch points on $[0,2 \pi)$. If $\bar{u}=\mu_{s}$, for some $s \in(0, \pi)$ or $s \in(\pi, 2 \pi)$, then the convergence of the integral in (14) implies that the two switch points of $\bar{u}_{\epsilon}$ on $[0,2 \pi)$ tend to the two switch points, $s$ and $s \pm \pi$, of $\bar{u}$ as $\epsilon \downarrow$ o. If $\bar{u}=\mu_{0}$ or $\bar{u}=\mu_{\pi}$, then the convergence of the integral in (14) implies that one switch point of $\bar{u}_{\epsilon}$ on $[0,2 \pi)$ tends to $\pi$ and the other switch point tends to either 0 or $2 \pi$ as $\epsilon \downarrow 0$. In Ref. 1 , it is proved that the conditions of Theorem 4.1 are satisfied for the linear system

$$
\ddot{x}+\epsilon \dot{x}+x=\epsilon u,
$$

the Duffing equation

$$
\ddot{x}+\epsilon\left(\dot{x}+x^{3}\right)+x=\epsilon u,
$$

and the Van der Pol equation

$$
\ddot{x}+\epsilon\left(x^{2}-1\right) \dot{x}+x=\epsilon u \text {. }
$$

We now finally interpret Theorem 4.1 for the Duffing equation. In this case, $\bar{r}$ must satisfy

$$
9 \pi^{2} \bar{r}^{6} / 16+\pi^{2} \bar{r}^{2}=16 .
$$


It follows that

$$
\vec{r}=1.011, \quad \alpha(\bar{r})=-3.175, \quad \beta(\bar{r})=2.433 .
$$

Consequently, $\bar{u}=\mu_{s}$, where $s$ satisfies

$$
4 \cos s=-3.175, \quad-4 \sin s=2.433 \text {. }
$$

It follows that

$$
s=3.795 \text {. }
$$

Summarizing, for the Duffing equation, the limit of the maximum amplitude equals 1.011 as $\epsilon \downarrow 0$; and the optimal bang-bang controls, for which the maximum amplitude is attained at $t=0$, have two switch points on $[0,2 \pi)$ for sufficiently small $\epsilon>0$, tending to 0.654 and 3.795 , respectively, as $\epsilon \downarrow 0$. Furthermore, around these two switch points, for increasing $t, u(t)$ changes from +1 to -1 and from -1 to +1 , respectively. We note that, from the synchronization theory, it follows that, for the control $\bar{u}=\mu_{s}$ itself, there also are $2 \pi$-periodic solutions with an amplitude tending to $\bar{r}$ as $\epsilon \downarrow 0$; however, they are not necessarily optimal.

\section{Linear Case}

We consider the linear differential equation

$$
\ddot{x}+\epsilon \dot{x}+x=\epsilon u
$$

and some modifications to illustrate the results of the previous sections.

Throughout this section, we assume that $\epsilon \in(0, \sqrt{3})$. We first determine, for arbitrary $\epsilon \in(0, \sqrt{3})$ and $u \in \Omega$, the initial values

$$
x_{\epsilon u}:=x(0, \epsilon, u), \quad \dot{x}_{\epsilon u}:=\dot{x}(0, \epsilon, u)
$$

of $2 \pi$-periodic solutions $x(t, \epsilon, u)$ of (15). Let

$$
\epsilon \in(0, \sqrt{3}), \quad u \in \Omega, \quad x_{0}, \dot{x}_{0} \in \mathbb{R} \text {. }
$$

Then, the solution $x(t)$ of $\mathrm{Eq}$. (15), with initial values

$$
x(0)=x_{0}, \quad \dot{x}(0)=\dot{x}_{0},
$$

reads

$$
\begin{aligned}
x(t) & =e^{-\frac{1}{2} \epsilon t}\left\{\left(\cos \left(\frac{1}{2} \beta_{\epsilon} t\right)+\epsilon \beta_{\epsilon}^{-1} \sin \left(\frac{1}{2} \beta_{\epsilon} t\right)\right) x_{0}+\left(2 \beta_{\epsilon}^{-1} \sin \left(\frac{1}{2} \beta_{\epsilon} t\right)\right) \dot{x}_{\theta}\right\} \\
& +2 \epsilon \beta_{\epsilon}^{-1} \int_{0}^{t} e^{-\frac{1}{2} \epsilon(t-\tau)} \sin \left[\frac{1}{2} \beta_{\epsilon}(t-\tau)\right] u(\tau) d \tau
\end{aligned}
$$


where

$$
\beta_{\epsilon}:=\left(4-\epsilon^{2}\right)^{1 / 2}
$$

The initial values $x_{0}, \dot{x}_{0}$ are the intial values of a $2 \pi$-periodic solution iff

$$
x(2 \pi)=x_{0}, \quad \dot{x}(2 \pi)=\dot{x}_{0} .
$$

Taking $t=2 \pi$ in (16), these two equations convert into two linear equations in the variables $x_{0}, \dot{x}_{0}$. It turns out that these equations have a unique solution. For $x_{0}$, there holds

$$
x_{0}=\epsilon \beta_{\epsilon}^{-1} e^{\pi \epsilon}\left(\cosh (\pi \epsilon)-\cos \left(\pi \beta_{\epsilon}\right)\right)^{-1} \int_{0}^{2 \pi t} h_{\epsilon}(\tau) u(\tau) d \tau
$$

where

$$
h_{\epsilon}(t)=e^{-\frac{1}{2} \epsilon(2 \pi-t)}\left\{\sin \left[\frac{1}{2} \beta_{\epsilon}(2 \pi-t)\right]+e^{-\pi \epsilon} \sin \left(\frac{1}{2} \beta_{\epsilon} t\right)\right\} .
$$

Hence, for every $\epsilon \in(0, \sqrt{3})$ and $u \in \Omega$, there is exactly one $2 \pi$-periodic solution $x(t, \epsilon, u)$, and the initial value $x_{\epsilon u}$ is given by (17). As we mentioned already in Section 1, the maximum amplitude $A_{\epsilon}$ equals the maximum initial value $\left|x_{\epsilon u}\right|$. Since, in the case of (15),

$$
f(x, \dot{x})=\dot{x}
$$

is odd, $A_{\epsilon}$ equals the maximum $x_{\epsilon u}$. From (17), it follows that $x_{\epsilon u}$ is maximized for the bang-bang control

$$
\tilde{u}_{\epsilon}(t):=\operatorname{sign}\left(h_{\epsilon}(t)\right) .
$$

One can show that, for every $\epsilon \in(0, \sqrt{3})$, the function $h_{\epsilon}$ has two zeros, $T_{1 \epsilon}$ and $T_{2 \epsilon}$ on $(0,2 \pi)$, satisfying

$$
T_{1 \epsilon}:=2 \pi-2 \beta_{\epsilon}^{-1}\left(M_{\epsilon}+\pi\right), \quad T_{2 \epsilon}:=2 \pi-2 \beta_{\epsilon}^{-1} M_{\epsilon},
$$

where

$$
M_{\epsilon}:=\arctan \left[\sin \left(\pi \beta_{\epsilon}\right) /\left(\cos \left(\pi \beta_{\epsilon}\right)-e^{\pi \epsilon}\right)\right] .
$$

Furthermore,

$$
\begin{array}{ll}
h_{\epsilon}(t)<0, & \text { on }\left(0, T_{1 \epsilon}\right) \cup\left(T_{2 \epsilon}, 2 \pi\right), \\
h_{\epsilon}(t)>0, & \text { on }\left(T_{1 \epsilon}, T_{2 \epsilon}\right) .
\end{array}
$$

Consequently, the bang-bang controls $u_{\epsilon}$ have two switch points, $T_{1 \epsilon}$ and $T_{2 \epsilon}$, on $(0,2 \pi)$, and

$$
\bar{u}_{\epsilon}(t)= \begin{cases}-1, & t \in\left[0, T_{1 \epsilon}\right) \cup\left[T_{2 \epsilon}, 2 \pi\right), \\ +1, & t \in\left[T_{1 \epsilon}, T_{2 \epsilon}\right) .\end{cases}
$$


Using this, (17), and (18), one can show by integration over three separate intervals that the maximum amplitude $x_{\epsilon \bar{u}_{\epsilon}}$ equals

$$
x_{\epsilon \tilde{u}_{\epsilon}}=-\epsilon\left(2 \sin M_{\epsilon}\left(e^{\frac{1}{2} \epsilon T_{1 \epsilon}}+e^{\frac{1}{2} \epsilon T_{2 \epsilon}}\right) /\left(\sin \left(\pi \beta_{\epsilon}\right)+1\right) .\right.
$$

It turns out that, for $\epsilon \downarrow 0$, we have

$$
T_{1 \epsilon} \uparrow \pi, \quad T_{2 \epsilon} \uparrow 2 \pi, \quad x_{\epsilon \bar{u}_{\epsilon}} \downarrow 4 / \pi,
$$

with

$$
\begin{aligned}
& T_{1 \epsilon}=\pi-\epsilon / 4+O\left(\epsilon^{3}\right), \\
& T_{2 \epsilon}=2 \pi-\epsilon / 4+\pi \epsilon^{2} / 8+O\left(\epsilon^{3}\right), \\
& x_{\epsilon \bar{u}_{\epsilon}}=4 / \pi+[\pi / 12-5 / 8 \pi] \epsilon^{2}+O\left(\epsilon^{3}\right) .
\end{aligned}
$$

Consequently, the limit of the maximum amplitude $A_{\varepsilon}$ is $4 / \pi$ as $\epsilon$ approaches zero, and the optimal controls $\bar{u}_{\epsilon}$ converge to $\bar{u}:=\mu_{\pi}$ in the sense of (14). We now deduce these results by applying the synchronization theory to Eq. (15). From the definitions in (7), we obtain, for the case

$$
f(x, \dot{x})=\dot{x},
$$

that

$$
\begin{aligned}
& \alpha(r)=-\int_{0}^{2 \pi} r \sin ^{2} t d t=-\pi r \\
& \beta(r)=-\int_{0}^{2 \pi} r \sin t \cos t d t=0 .
\end{aligned}
$$

Consequently, for $\bar{r}$ defined by (11), there must hold

$$
\pi^{2} \bar{r}^{2}=16
$$

hence,

$$
\bar{r}=4 / \pi
$$

Since

$$
\left(\pi^{2} r^{2}\right)^{\prime}=2 \pi^{2} r \neq 0, \quad r=\bar{r},
$$

it follows from (i) of Theorem 4.1 that the limit of the maximum amplitude is, indeed, $4 / \pi$. Since

$$
(\alpha(\bar{r}), \beta(\bar{r}))=(-4,0),
$$

the control $\bar{u}$ defined by (13) is $\bar{u}=\mu_{\pi}$, and then the foregoing convergence result for the optimal controls $\bar{u}_{\varepsilon}$ indeed coincides with (14) of Theorem 4.1. 
Remark 5.1. If we add the even function

$$
g(x, \dot{x}):=a, \quad a \in \mathbb{R},
$$

to the function

$$
f(x, \dot{x})=\dot{x},
$$

i.e., if we consider the linear differential equation

$$
\ddot{x}+\epsilon(a+\dot{x})+x=\epsilon u,
$$

then, obviously,

$$
x_{a}(t, \epsilon, u):=x(t, \epsilon, u)-\epsilon a
$$

is a $2 \pi$-periodic solution of (22), where, as before, $x(t, \epsilon, u)$ is the $2 \pi$ periodic solution of (15). From the uniqueness of $2 \pi$-periodic solutions of (15), it follows that this is the only $2 \pi$-periodic solution of (22) for fixed

$$
a \in \mathbb{R}, \quad u \in \Omega, \quad \epsilon>0 .
$$

Obviously, $x_{a}(t, \epsilon, u)$ does depend on $a$, but $\lim _{\epsilon \downarrow 0} x_{\alpha}(t, \epsilon, u)$ does not. This illustrates the final statement of Remark 3.3. Maximizing the initial value

$$
\left|x_{a \epsilon u}\right|:=\left|x_{a}(0, \epsilon, u)\right|
$$

means maximizing

$$
\left|\epsilon \beta_{\epsilon}^{-1} e^{\pi \epsilon}\left[\cosh (\pi \epsilon)-\cos \left(\pi \beta_{\epsilon}\right)\right]^{-1} \int_{0}^{2 \pi} h_{\epsilon}(\tau) u(\tau) d t-\epsilon a\right| .
$$

Hence, the maximizing controls $\bar{u}_{a \epsilon}$ must satisfy

$$
\begin{aligned}
& \vec{u}_{a \epsilon}(t)=\operatorname{sign}\left(h_{\epsilon}(t)\right), \quad \text { if } a<0, \\
& \bar{u}_{a \epsilon}(t)=-\operatorname{sign}\left(h_{\varepsilon}(t)\right), \quad \text { if } a>0 .
\end{aligned}
$$

Thus, for $a<0$,

$$
\bar{u}_{a \epsilon}=\bar{u}_{\epsilon}
$$

and, for $a>0$,

$$
\bar{u}_{a \epsilon}=-\bar{u}_{\epsilon},
$$

where $\bar{u}_{\epsilon}$ is given by (20). Consequently [cf. formula (19)], as in the case of (15), the optimal controls are bang-bang controls and have two switch points on $(0,2 \pi)$; for $a<0$, these optimal controls converge to $\bar{u}=\mu_{\pi}$; for $a>0$, these optimal controls converge to $\bar{u}=-\mu_{\pi}$ in the sense of (14). On the other hand, for both cases, $a<0$ and $a>0$, we have, for the limit of 
the maximum amplitude,

$$
\lim _{\epsilon \downarrow 0}\left|x_{a}\left(0, \epsilon, \bar{u}_{a \epsilon}\right)\right|=\lim _{\epsilon \downarrow 0}\left|x\left(0, \epsilon, \bar{u}_{a \epsilon}\right)\right|=\lim _{\epsilon \downarrow 0}\left|x\left(0, \epsilon, \tilde{u}_{\epsilon}\right)\right|=4 / \pi
$$

We observe that the control $\tilde{u}=\mu_{\pi}$ has synchronization point $(+\vec{r}, 0)$, whereas the control $\bar{u}=-\mu_{r}$ has synchronization point $(-\bar{r}, 0)$. So, this example shows that, in the case of $f$ containing a nonzero even part, there are two (inverse) candidates for the limit $\bar{u}$ of the optimal controls in the sense of (14), characterized by the fact that they correspond to the synchronization points $(+\bar{r}, 0)$ and $(-\bar{r}, 0)$, where $\bar{r}$ is defined by $(11)$. The synchronization theory of the previous sections gives no decisive answer on the question of which of the two candidates is the right one. However, the limit of the maximum amplitude is $\bar{r}$, not depending on the nonzero even part of $f$.

Remark 5.2. For the linear differential equation

$$
\ddot{x}+\epsilon a+x=\epsilon u
$$

where

$$
f(x, \dot{x})=a, \quad a \in \mathbb{R},
$$

is an even function, the general solution with initial values

$$
x(0)=x_{0}, \quad \dot{x}(0)=\dot{x}_{0},
$$

reads

$$
x(t)=x_{0} \cos t+\dot{x}_{0} \sin t+\epsilon \int_{0}^{t} \sin (t-\tau)(u(\tau)-a) d \tau
$$

The conditions

$$
x(0)=x(2 \pi), \quad \dot{x}(0)=\dot{x}(2 \pi)
$$

now yield the two linear equations

$$
\begin{aligned}
& x_{0}=x_{0}-\epsilon \int_{0}^{2 \pi}(u(\tau)-a) \sin \tau d \tau \\
& \dot{x}_{0}=\dot{x}_{0}+\epsilon \int_{0}^{2 \pi}(u(\tau)-a) \cos \tau d \tau
\end{aligned}
$$

Hence, if

$$
\int_{0}^{2 \pi} \sin \tau u(\tau) d \tau=0=\int_{0}^{2 \pi} \cos \tau u(\tau) d \tau
$$


for the control $u \in \Omega$, then every solution of (23) is $2 \pi$-periodic. Otherwise, there are no $2 \pi$-periodic solutions of (23) for the control $u$. Consequently, for Eq. (23) the AMP has no solution.

\section{Van der Pol Equation}

We apply the synchronization theory to the Van der Pol equation

$$
\ddot{x}+\epsilon\left(x^{2}-1\right) \dot{x}+x=\epsilon u \text {. }
$$

The function

$$
f(x, \dot{x})=\left(x^{2}-1\right) \dot{x}
$$

is odd. According to the definitions in (7), we have

$$
\begin{aligned}
& \alpha(r)=\int_{0}^{2 \pi}\left(1-r^{2} \cos ^{2} t\right) r \sin ^{2} t d t=\pi r\left(1-r^{2} / 4\right), \\
& \beta(r)=\int_{0}^{2 \pi}\left(1-r^{2} \cos ^{2} t\right) r \sin t \cos t d t=0 .
\end{aligned}
$$

The function $\alpha(r)$ is odd, has a local maximum $4 \pi \sqrt{3} / 9$ at $r=2 / \sqrt{3}$, and is zero at $r=0$ and $r=2$. Furthermore,

$$
\alpha(r) \rightarrow-\infty, \quad r \rightarrow \infty .
$$

The synchronization curve

$$
\operatorname{sc}(r)=(\alpha(r), \beta(r))
$$

follows the trajectory reflected in Fig. 2 . Note that

$$
4 \pi \sqrt{3} / 9 \approx 2.418, \quad 2 / \sqrt{3} \approx 1.155 \text {. }
$$

The point $(4 \pi \sqrt{3} / 9,0)$ is the point for which

$$
r=-4 / \sqrt{3} \text { or } r=2 / \sqrt{3} \text {. }
$$

For all values of $r$, except $-2 / \sqrt{3}, 2 / \sqrt{3},-2,2$, the synchronization curve is not orthogonal to the radius. Consequently, for points $(r, \phi)$ which satisfy the synchronization equations and for which $r$ is not equal to $-2 / \sqrt{3}$, $2 / \sqrt{3},-2,2$, we have

$$
\operatorname{det} F_{u, x}(r, \phi) \neq 0 \text {. }
$$

This agrees indeed with [cf. formula (10)]

$$
\operatorname{det} F_{u, x}(r, \phi)=\left(8 \pi^{2} r\right)^{-1}\left(\alpha^{2}(r)+\beta^{2}(r)^{\prime}\right)=\left(4-r^{2}\right)\left(4-3 r^{2}\right) / 64 \text {. }
$$


From Fig. 2, it follows that, for controls $u \in \Omega$, for which

$$
0<\alpha_{u}^{2}+\beta_{u}^{2}<16 \pi^{2} / 27,
$$

there are at least three $2 \pi$-periodic solutions for small $\epsilon>0$, and the limiting value of the corresponding amplitudes is less than $4 / \sqrt{3}$. For controls $u \in \Omega$, for which

$$
\alpha_{u}^{2}+\beta_{u}^{2} \geq 16 \pi^{2} / 27,
$$

there is at least one $2 \pi$-periodic solution for small $\epsilon>0$, and the limiting value of the amplitude is greater than $4 / \sqrt{3}$. For $\bar{r}$ defined by (11), clearly

$$
\bar{r}^{3}-4 \bar{r}-16 / \pi=0
$$

holds. It turns out that the only real solution is

$$
\bar{r} \approx 2.4632459 \text {. }
$$

The bang-banger $\bar{u}$ of (iii) of Theorem 4.1 must satisfy

$$
\left(\alpha_{\bar{u}}, \beta_{\bar{u}}\right)=(-4,0) \text {, }
$$

which implies

$$
\bar{u}=\mu_{\pi}
$$

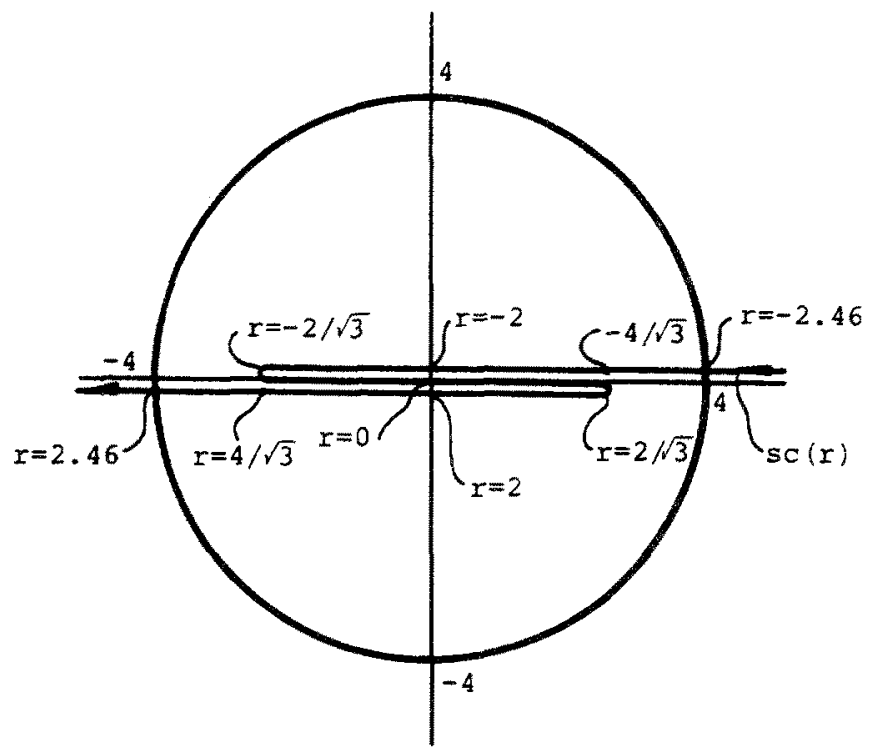

Fig. 2. Synchronization curve for the Van der Pol equation. 
Table 1. Numerical results for the Van der Pol equation.

\begin{tabular}{cccccccc}
\hline$\epsilon$ & $A_{\epsilon}$ & $T_{1 \epsilon}$ & $T_{2 \epsilon}$ & $\epsilon$ & $A_{\epsilon}$ & $T_{1 \epsilon}$ & $T_{2 \varepsilon}$ \\
\hline 0.005 & 2.46324 & 3.141409 & 6.282848 & 0.500 & 2.45450 & 3.103538 & 6.281387 \\
0.010 & 2.46324 & 3.141169 & 6.282550 & 0.600 & 2.45089 & 3.099759 & 6.282134 \\
0.020 & 2.46323 & 3.140480 & 6.282171 & 0.700 & 2.44681 & 3.098152 & 6.282594 \\
0.030 & 2.46321 & 3.139874 & 6.281726 & 0.800 & 2.44232 & 3.098304 & 6.282872 \\
0.040 & 2.46318 & 3.139248 & 6.281310 & 0.900 & 2.43753 & 3.099876 & 6.282987 \\
0.050 & 2.46315 & 3.138602 & 6.280922 & 1.000 & 2.43251 & 3.102226 & 6.283080 \\
0.100 & 2.46288 & 3.135016 & 6.279471 & 1.300 & 2.41689 & 3.110826 & 6.283107 \\
0.130 & 2.46263 & 3.132630 & 6.278940 & 1.600 & 2.40153 & 3.114540 & 6.283135 \\
0.170 & 2.46219 & 3.129223 & 6.278585 & 2.000 & 2.38306 & 3.094372 & 6.283160 \\
0.200 & 2.46179 & 3.126554 & 6.278534 & 2.100 & 2.37900 & 3.078114 & 6.283167 \\
0.300 & 2.46000 & 3.117592 & 6.279184 & 2.200 & 2.37526 & 3.050149 & 6.283173 \\
0.400 & 2.45756 & 3.109598 & 6.280338 & 2.300 & 2.37202 & 2.983813 & 6.283179 \\
\hline
\end{tabular}

Thus, the maximum amplitude $A_{\varepsilon}$ tends to $\bar{r}$ as $\epsilon \downarrow 0$. Moreover, for sufficiently small $\epsilon>0$, the optimal controls are bang-bang controls with two switch points on $[0,2 \pi)$; and the optimal controls $\bar{u}_{\epsilon} \in \Omega$ for which there exists a $2 \pi$-periodic solution with the maximum amplitude attained at $t=0$ converge to $\ddot{u}=\mu_{\pi}$ in the sense of (14) as $\epsilon \downarrow 0$. Therefore, one switch point of $\bar{u}_{\epsilon}$ tends to $\pi$ and the other tends to either 0 or $2 \pi$ as $\epsilon \downarrow 0$. Regarding the results for the linear system (15), we may expect the other switch point to tend to $2 \pi$. Furthermore,

$$
\bar{u}_{\epsilon}(t) \rightarrow-1, \quad t \uparrow 2 \pi .
$$

In order to verify the foregoing analytic results concerning the limit of $A_{\varepsilon}$ and the convergence of $\bar{u}_{e}$, the IVMP has been solved numerically for some values of $\epsilon$ in the interval $(0,2.3]$; for details, we refer to Ref. 1. The results are given in Table 1. The columns denoted by $A_{\epsilon}, T_{1 \epsilon}, T_{2 \epsilon}$ indicate, respectively, the computed value of the maximum amplitude, the first switch point on $(0,2 \pi)$, and the second switch point on $(0,2 \pi)$ of $\bar{u}_{\varepsilon}$. The numerical results agree with the analytic results. Obviously,

$$
A_{\epsilon}=2.4632459+O\left(\epsilon^{2}\right), \quad \epsilon \downarrow 0 ;
$$

hence, the coefficient of the term of order one seems to be zero, as it is the case for the linear system (15).

\section{Appendix}

Here, we will prove the statements (i), (ii), (iv) of Theorem 4.1. The statement (iii) follows immediately from the proof of Theorem 3.1 . 
In this appendix, we do not distinguish explicitly between points in $\mathbb{R}^{n}$ and column vectors in $\mathbb{R}^{n}$. By $|x|$, we mean the Euclidean norm (or Euclidean distance to zero) of the vector or point $x \in \mathbb{R}^{n}$. We extend the set of admissible controls,

$\Omega^{\prime}:=\{\mathrm{u}: \mathbb{R} \rightarrow[-1,+1] \mid u$ is $2 \pi$-periodic and measurable on $(0,2 \pi)\}$.

7.1. Existence of Optimal Measurable Controls for the AMP. As in Theorem 4.1, we assume that the initial values

$$
(x(0), \dot{x}(0))=: x_{0} \in \mathbb{R}^{2}
$$

of $2 \pi$-periodic solutions of the differential equation (1) are uniformly bounded, say $\left|x_{0}\right| \leq m$, for all controls $u \in \Omega^{\prime}$ and sufficiently small $\epsilon>0$, say $0<\epsilon<\epsilon_{m}$. By $x_{u}\left(t, t_{0}, x_{0}, \epsilon\right)$, we mean the 2-dimensional vector solution of the 2-dimensional system corresponding to (1), for control $u \in \Omega^{\prime}$, parameter value $\epsilon \geq 0$, and initial values

$$
x_{u}\left(t_{0}, t_{0}, x_{0}, \epsilon\right)=x_{0} \in \mathbb{R}^{2} .
$$

If $\epsilon=0$ and $u \in \Omega$, then the solution $x_{u}\left(t, 0, x_{0}, 0\right)$ exists on the whole interval $[0,2 \pi)]$, for each $x_{0} \in \mathbb{R}^{2}$. Retracing the proofs of the standard results (Theorem 7.4, Chapter 1 and Theorem 4.1, Chapter 2, Ref. 5) concerning the dependence of the solutions of differential equations on the initial condition and parameters, one can show that there exists an $\epsilon_{f}$, not depending on $u \in \Omega^{\prime}$ and

$$
x_{0} \in X_{m}:=\left\{x_{0} \in \mathbb{R}^{2}|| x_{0} \mid \leq m\right\},
$$

but only on $f$, such that $x_{u}\left(t, 0, x_{0}, \epsilon\right)$ exists on $[0,2 \pi]$ for all

$$
0 \leq \epsilon<\epsilon_{f}, \quad u \in \Omega^{\prime}, \quad x_{0} \in X_{m} .
$$

From Theorem 3.1 and Remark 3.2 of Ref. 6, it then follows that

$$
W_{\epsilon}\left(x_{0}\right):=\left\{x_{u}\left(2 \pi, 0, x_{0}, \epsilon\right) \mid u \in \Omega^{\prime}\right\}
$$

is compact for every $0<\epsilon<\epsilon_{f}$ and each $x_{0} \in X_{m}$. The convexity condition in Theorem 3.1 of Ref. 6 is fulfilled, since (1) is linear in $u$. We are now ready to prove an existence theorem.

Theorem 7.1. Under the condition of Theorem 4.1, the maximum amplitude $A_{\epsilon}$ of the AMP exists for differential equation (1) for sufficiently small $\epsilon>0$, if the set of admissible controls is $\Omega^{\prime}$.

Proof. In view of the definition of $X_{m}$ and the equivalence of the AMP and the IVMP, it suffices to show that, for

$$
0<\epsilon<\min \left(\epsilon_{m}, \epsilon_{f}\right),
$$


the set

$$
V_{\epsilon}:=\left\{x_{0} \in X \mid x_{0} \in W_{\epsilon}\left(x_{0}\right)\right\}
$$

is compact. Let

$$
0<\epsilon<\min \left(\epsilon_{m}, \epsilon_{f}\right)
$$

Obviously, $V_{\epsilon}$ is bounded. We show that $V_{\epsilon}$ is closed. Let

$$
x_{k} \in V_{\varepsilon}, \quad x_{k} \rightarrow \bar{x}, \quad k \rightarrow \infty .
$$

If $\mathrm{d}(\cdot, \cdot)$ denotes the Hausdorff distance of two nonempty compact subsets of $\mathbb{R}^{2}$, then we have

$$
\begin{aligned}
\min _{y \in W_{\epsilon}(\bar{x})}|\bar{x}-y| & \leq\left|\bar{x}-x_{k}\right|+\min _{y \in W_{\epsilon}(\bar{x})}\left|x_{k}-y\right| \\
& \leq\left|\bar{x}-x_{k}\right|+\mathrm{d}\left(W_{\epsilon}\left(x_{k}\right), W_{\epsilon}(\bar{x})\right) \rightarrow 0, \quad k \rightarrow \infty,
\end{aligned}
$$

since the map $x_{0} \rightarrow W_{\epsilon}\left(x_{0}\right)$ is continuous with respect to the Hausdorff distance (cf. Ref. 7, Theorem 3.3). Hence, $\bar{x} \in W_{\epsilon}(\bar{x})$, and thus $W_{\epsilon}(\tilde{x})$ is closed.

7.2. Necessary Conditions for Optimal Controls. The IVMP for the differential equation (1) can be reformulated as follows: determine a control $u \in \Omega^{\prime}$ such that the differential system and the boundary conditions

$$
\begin{array}{ll}
\dot{x}_{1}=x_{2}, & x_{1}(0)=y_{1}(0), \\
\dot{x}_{2}=-\epsilon f\left(x_{1}, x_{2}\right)-x_{1}+\epsilon u, & x_{2}(0)=y_{2}(0), \\
\dot{y}_{1}=0, & x_{1}(2 \pi)=y_{1}(2 \pi), \\
\dot{y}_{2}=0, & x_{2}(2 \pi)=y_{2}(2 \pi),
\end{array}
$$

have a solution, such that $x_{1}(2 \pi)$ is maximal. This is a continuous-time optimal control problem, described in Section 5 of Ref. 8 , with

$$
\begin{aligned}
& T:=2 \pi, \quad n:=4, \quad U:=[-1,+1], \\
& f\left(x_{1}, x_{2}, y_{1}, y_{2}, u\right):=\left(x_{2},-\epsilon f\left(x_{1}, x_{2}\right)-x_{1}-\epsilon u, 0,0\right), \\
& X_{0}:=X_{1}:=\left\{\left(x_{1}, x_{2}, y_{1}, y_{2}\right) \in \mathbb{R}^{4} \mid\left(x_{1}, x_{2}\right)=\left(y_{1}, y_{2}\right)\right\}, \\
& h\left(x_{1}, x_{2}, y_{1}, y_{2}\right):=x_{1} .
\end{aligned}
$$

From the version of Pontryagin's maximum principle for this problem, derived in Ref. 8, we obtain the following result. 
Theorem 7.2. If $\bar{u} \in \Omega^{\prime}$ and $x$ is an optimal solution of the IVMP, then there exists a number $\rho \geq 0$ and a function $\psi:[0,2 \pi] \rightarrow \mathbb{R}$, solution of the differential equation

$$
\ddot{\psi}+\psi=\epsilon\left\{\psi\left[(d / d t) f_{x_{2}}\left(x_{1}, x_{2}\right)-f_{x_{1}}\left(x_{1}, x_{2}\right)\right]+\dot{\psi} f_{x_{2}}\left(x_{1}, x_{2}\right)\right\},
$$

satisfying the boundary conditions

$$
\psi(0)=\psi(2 \pi), \quad \dot{\psi}(0)=\dot{\psi}(2 \pi)+\rho,
$$

such that

(i) $\quad(\rho, \psi(0), \dot{\psi}(0)) \neq 0$;

(ii) $\ddot{x}+x=\epsilon(\bar{u}-f(x, \dot{x})), x(0)=x(2 \pi), \dot{x}(0)=\dot{x}(2 \pi)$;

(iii) $\bar{u}(t)=\operatorname{sign}(\psi(t))$ on $[0,2 \pi]$, almost everywhere.

Proof. We apply Theorem 5.15 of Ref. 8 to Eqs. (24) for the case of measurable controls (cf. Appendix B of Ref. 8). At an arbitrary point $b \in X_{0}$, $X_{1}$, the cones

$$
E_{0}:=E_{1}:=\left\{\left(p_{1}, p_{2}, q_{1}, q_{2}\right) \mid\left(p_{1}, p_{2}\right)=\left(q_{1}, q_{2}\right)\right\}
$$

are derived cones. The polar cones are

$$
E_{0}^{0}=E_{1}^{0}=\left\{\left(v_{1}, v_{2}, w_{1}, w_{2}\right) \mid\left(v_{1}, v_{2}\right)=-\left(w_{1}, w_{2}\right)\right\} .
$$

Consequently, Theorem 5.15 guarantees that, if $\bar{u} \in \Omega^{\prime},\left(x_{1}, x_{2}, y_{1}, y_{2}\right)$ is an optimal solution of the problem given by (24), then there exists a function

$$
\tilde{\psi}:[0,2 \pi] \rightarrow \mathbb{R}^{4}\left(\tilde{\psi}(t)=\psi_{1}(t), \psi_{2}(t), \chi_{1}(t), \chi_{2}(t)\right),
$$

a vector

$$
\tilde{\phi} \in \mathbb{R}^{4}, \quad \tilde{\phi}=\left(\phi_{1}, \phi_{2}, \lambda_{1}, \lambda_{2}\right),
$$

and a number $\tilde{\rho} \geq 0$, such that ${ }^{3}$

$$
\begin{array}{ll}
\left(\tilde{\rho}, \psi_{1}(2 \pi), \psi_{2}(2 \pi), \chi_{1}(2 \pi),\right. & \left.\chi_{2}(2 \pi), \phi_{1}, \phi_{2}, \lambda_{1}, \lambda_{2}\right) \neq 0, \\
\dot{\psi}_{1}=\psi_{2}+\epsilon \psi_{2} f_{x_{1}}\left(x_{1}, x_{2}\right), & \dot{\chi}_{1}=0, \\
\dot{\psi}_{2}=-\psi_{1}+\epsilon \psi_{2} f_{x_{2}}\left(x_{1}, x_{2}\right), & \dot{\chi}_{2}=0, \\
\psi_{1}(0)=-\chi_{1}(0), & \phi_{1}=-\lambda_{1}, \\
\psi_{2}(0)=-\chi_{2}(0), & \phi_{2}=-\lambda_{2}, \\
\psi_{1}(2 \pi)=\tilde{\rho}-\phi_{1}, & \psi_{2}(2 \pi)=-\phi_{2}, \\
\chi_{1}(2 \pi)=-\lambda_{1}, & \chi_{2}(2 \pi)=-\lambda_{2},
\end{array}
$$

\footnotetext{
${ }^{3}$ Relation (26a) is called the nontriviality condition.
} 
and, almost everywhere on $[0,2 \pi]$,

$$
\begin{aligned}
\psi_{1} x_{2} & +\psi_{2}\left(-\epsilon f\left(x_{1}, x_{2}\right)-x_{1}+\epsilon \bar{u}\right) \\
& =\max _{v \in[-1,+1]} \psi_{1} x_{2}+\psi_{2}\left(-\epsilon f\left(x_{1}, x_{2}\right)-x_{1}+\epsilon v\right) .
\end{aligned}
$$

We will show that the number $\tilde{\rho}$ and the function $\psi_{2}$ satisfy the statements of Theorem 7.2. Differentiating the differential equation for $\psi_{2}$ and substituting the differential equation for $\psi_{1}$ yields indeed (25) for $\psi_{2}$. We have

$$
\begin{aligned}
& \psi_{1}(2 \pi)=\tilde{\rho}-\phi_{1}=\tilde{\rho}+\lambda_{1}=\tilde{\rho}-\chi_{1}(2 \pi)=\tilde{\rho}-\chi_{1}(0)=\tilde{\rho}+\psi_{1}(0) \\
& \psi_{2}(2 \pi)=-\phi_{2}=\lambda_{2}=-\chi_{2}(2 \pi)=-\chi_{2}(0)=\psi_{2}(0)
\end{aligned}
$$

Consequently,

$$
\begin{aligned}
\dot{\psi}_{2}(0) & =-\psi_{1}(0)+\epsilon \psi_{2}(0) f_{x_{2}}\left(x_{1}(0), x_{2}(0)\right) \\
& =\tilde{\rho}-\psi_{1}(2 \pi)+\epsilon \psi_{2}(2 \pi) f_{x_{2}}\left(x_{1}(2 \pi), x_{2}(2 \pi)\right)=\tilde{\rho}+\dot{\psi}_{2}(2 \pi) .
\end{aligned}
$$

So, $\psi_{2}$ satisfies both the boundary conditions. Since

$$
\phi_{1}=-\lambda_{1}=\chi_{1}(2 \pi)=\chi_{1}(0)=-\psi_{1}(0)
$$

and, similarly,

$$
\phi_{2}=-\psi_{2}(0),
$$

it follows from the nontriviality condition (26a) that

$$
\left(\tilde{\rho}, \psi_{2}(0), \dot{\psi}_{2}(0)\right) \neq 0
$$

Finally,

$$
\epsilon \psi_{2} \bar{u}=\max _{v \in[-1,+1]} \epsilon \psi_{2} v
$$

almost everywhere on $[0,2 \pi]$, which implies

$$
\bar{u}(t)=\operatorname{sign}\left(\psi_{2}(t)\right), \quad \text { on }[0,2 \pi],
$$

almost everywhere.

7.3. Number of Switch Points for Optimal Controls. According to Theorem 7.2, a point $\tau \in[0,2 \pi)$ can only be a switch point of the optimal bang-bang control $\bar{u} \in \Omega^{\prime}$ if $\psi(\tau)=0$, where $\psi$ satisfies (25). If $\psi(t)$ has a finite number of zeros on $[0,2 \pi)$, then there exists an optimal piecewise continuous bang-bang control $\bar{u} \in \Omega$. For small $\epsilon>0$, we can give an upper bound for the number of zeros on $[0,2 \pi)$ of nontrivial solution of differential equations of the type (25). This is based on the following result. 
Lemma 7.1. The distance between two adjacent zeros of a nontrivial solution of the differential equation

$$
\ddot{x}+w \dot{x}+(1+v) x=0,
$$

where $w(t)$ and $v(t)$ are two continuous function satisfying

$$
|w(t)| \leq \mu, \quad|v(t)| \leq \mu, \quad \mu \geq 0,
$$

is at least $\pi /(1+2 \mu)$.

Proof. Without loss of generality, we assume

$$
x(0)=0, \quad \dot{x}(0)=1 .
$$

Let $T>0$ be the smallest number for which

$$
x(T)=0 .
$$

Obviously,

$$
\dot{x}(T)<0 \text {. }
$$

We consider the transformation

$$
x(t)=r(t) \cos (t+\phi(t)), \quad \dot{x}(t)=-r(t) \sin (t+\phi(t)) .
$$

In terms of the new variables $r(t)$ and $\phi(t)$, we obtain the system

$$
\begin{aligned}
& \dot{r}=r(v \cos (t+\phi)-w \sin (t+\phi)) \sin (t+\phi), \\
& \dot{\phi}=(v \cos (t+\phi)-w \sin (t+\phi)) \cos (t+\phi),
\end{aligned}
$$

and the initial conditions

$$
r(0)=1, \quad \phi(0)=-\pi / 2 .
$$

Consequently, for all $0 \leq t \leq T$,

$$
|\dot{\phi}(t)| \leq 2 \mu
$$

hence,

$$
|\phi(t)+\pi / 2| \leq 2 \mu t .
$$

On $(0, T)$, we have

$$
x(t)>0
$$

hence,

$$
|r(t)|>0 .
$$


Since

$$
r(0)=1>0,
$$

we have

$$
r(t)>0, \quad \text { on }(0, T) .
$$

Since furthermore

$$
x(t)=r(t) \cos (t+\phi(t))>0, \quad \text { on }(0, T),
$$

and

$$
0+\phi(0)=-\pi / 2,
$$

it follows that

$$
|t+\phi(t)|<\pi / 2, \quad \text { on }(0, T) .
$$

Consequently,

$$
T+\phi(T)= \pm \pi / 2 .
$$

Since

$$
\dot{x}(T)=-r(T) \sin (T+\phi(T))<0, \quad r(T)>0,
$$

it follows that

$$
T+\phi(T)=\pi / 2 \text {. }
$$

So, finally we obtain

$$
T=\pi / 2-\phi(T)>\pi / 2-(-\pi / 2+2 \mu T)=\pi-2 \mu T .
$$

The differential equation (25) can be written as

$$
\ddot{\psi}+w \dot{\psi}+(1+v) \psi=0,
$$

where

$$
\begin{aligned}
& v(t):=\epsilon\left[f_{x_{1}}\left(x_{1}(t), x_{2}(t)\right)-(d / d t)\left(f_{x_{2}}\left(x_{1}(t), x_{2}(t)\right)\right)\right], \\
& w(t):=-\epsilon\left(f_{x_{2}}\right)\left(x_{1}(t), x_{2}(t)\right) .
\end{aligned}
$$

The optimal $2 \pi$-periodic solution $x_{1}(t), x_{2}(t)$ is uniformly bounded for small $\epsilon>0$, and so are $v(t) / \epsilon$ and $w(t) / \epsilon$. Hence, for sufficiently small $\epsilon>0$, certainly

$$
|v(t)|<1 / 8, \quad|w(t)|<1 / 8 ;
$$

then, from Lemma 7.1, it follows that the distance of two zeros of $\psi(t)$ is at least $4 \pi / 5$. Consequently, for sufficiently small $\epsilon>0, \psi(t)$ has at most 
three zeros on $[0,2 \pi)$; then, $\bar{u}(t)$ has at most three switch points on $[0,2 \pi)$. Since a $2 \pi$-periodic bang-bang control has an even number of switch points on $[0,2 \pi)$, it follows that, for sufficiently small $\epsilon>0$, the optimal control is a bang-bang control $\bar{u}_{\epsilon} \in \Omega$ with zero or two switch points on $[0,2 \pi)$.

\subsection{Limit of the Maximum Amplitude and Limiting Behavior of Optimal} Controls as $\epsilon \downarrow 0$. In Section 2, we introduced the polar coordinates $r(t)$, $\phi(t)$, given by (3), to describe the solution of the differential equation (1). The periodicity conditions

$$
x(0)=x(2 \pi), \quad \dot{x}(0)=\dot{x}(2 \pi)
$$

were replaced by

$$
r(0)=r(2 \pi), \quad \phi(0)=\phi(2 \pi) .
$$

A solution $r(t), \phi(t)$ of (4), satisfying

$$
r(0)=r(2 \pi), \quad \phi(0)=\phi(2 \pi)+2 k \pi,
$$

for some $k \in \mathbb{Z}$, also yields a $2 \pi$-periodic solution of (1). For sufficiently small $\epsilon>0$, however, the optimal $2 \pi$-periodic solutions with a maximum initial value $A_{\epsilon}$ satisfy

$$
\phi(0)=\phi(2 \pi),
$$

as we will show now. Since $f$ is odd and continuous,

$$
f(0,0)=0 ;
$$

hence, there exists an $a>0$ such that

$$
|u(t)|:=|f(a \cos t,-a \sin t)| \leq 1, \quad t \in \mathbb{R} .
$$

For this control $u \in \Omega$,

$$
(x(t), \dot{x}(t)):=(a \cos t,-a \sin t)
$$

is a $2 \pi$-periodic solution of (1) for all $\epsilon>0$. Consequently, for an optimal $2 \pi$-periodic solution of (1), the maximum initial value of $r(0)$ satisfies $r(0) \geq a$, for all $\epsilon>0$. Obviously, $a \leq m$. If $\epsilon=0$, then

$$
r(t) \equiv r, \quad \phi(t) \equiv \phi
$$

is the solution of (4) on $[0,2 \pi]$ for any control $u \in \Omega$ and initial values

$$
r(0)=r>0, \quad \phi(0)=\phi \in \mathbb{R} .
$$

From standard proofs of uniform convergence of solutions of (4) with respect to the parameter $\epsilon$, it then follows that, for all $\mu>0$, there exists 
an $\epsilon_{\mu}$ such that, for all

$$
0 \leq \epsilon \leq \epsilon_{\mu}, \quad u \in \Omega, \quad a \leq r \leq m, \quad 0 \leq \phi \leq 2 \pi,
$$

the solution $r(t), \phi(t)$ of (4), with initial values

$$
r(0)=r, \quad \phi(0)=\phi,
$$

exists on $[0,2 \pi]$ and satisfies

$$
|r(t)-r|<\mu, \quad|\phi(t)-\phi|<\mu, \quad \text { on }[0,2 \pi] .
$$

Taking $\mu<2 \pi$, it follows that, for sufficiently small $\epsilon>0$, the optimal $2 \pi$-periodic solution of (1) with maximum initial value satisfies

$$
\phi(0)=\phi(2 \pi)
$$

Hence, we have showed that we may concentrate on $2 \pi$-periodic solutions of (4), for sufficiently small $\epsilon>0$. Taking $\mu=a / 2$, it follows that there exists an $\epsilon_{a}>0$ such that, for all

$$
0 \leq \epsilon \leq \epsilon_{a}, \quad u \in \Omega, \quad a \leq r \leq m, \quad 0 \leq \phi \leq 2 \pi,
$$

the solution $r(t), \phi(t)$ of (4), with initial values

$$
r(0)=r, \quad \phi(0)=\phi,
$$

exists on $[0,2 \pi]$ and satisfies

$$
r(t) \geq a / 2>0, \quad \text { on }[0,2 \pi] \text {. }
$$

Hence, for all $u \in \Omega$ and

$$
(r, \phi, \epsilon) \in D_{a}:=\left\{(r, \phi, \epsilon) \in \mathbb{R}^{3} \mid a \leq r \leq m, 0 \leq \phi \leq 2 \pi, 0 \leq \epsilon \leq \epsilon_{a}\right\},
$$

the function

$$
H_{u}(r, \phi, \epsilon):\left[\begin{array}{l}
\int_{0}^{2 \pi}[f(r(t) \cos (t+\phi(t)),-r(t) \sin (t+\phi(t))) \\
-u(t)] \sin (t+\phi(t)) \\
\int_{0}^{2 \pi} r(t)^{-1}[f(r(t) \cos (t+\phi(t)),-r(t) \sin (t+\phi(t))) \\
-u(t)] \cos (t+\phi(t))
\end{array}\right]
$$

is well defined. Here, $r(t), \phi(t)$ is the solution of (4) with control $u$, parameter value $\epsilon$, and initial values

$$
r(0)=r, \quad \phi(0)=\phi .
$$

We introduce the function $H_{u}$ for the following reason. If the initial values 
$a \leq r \leq m$ and $0 \leq \phi \leq 2 \pi$ correspond to a $2 \pi$-periodic solution of (4), then

$$
\begin{aligned}
(0,0) & =(r(2 \pi)-r, \phi(2 \pi)-\phi)=\left(\int_{0}^{2 \pi} \dot{r}(t) d t, \int_{0}^{2 \pi} \dot{\phi}(t) d t\right) \\
& =\epsilon H_{u}(r, \phi, \epsilon),
\end{aligned}
$$

and vice versa. So, the system (4), with control $u \in \Omega$ and parameter value $0<\epsilon \leq \epsilon_{a}$, admits a $2 \pi$-periodic solution with initial values

$$
a \leq r(0)=r \leq m, \quad 0 \leq \phi(0)=\phi \leq 2 \pi,
$$

iff

$$
H_{u}(r, \phi, \epsilon)=0 .
$$

We note that

$$
H_{u}(r, \phi, 0)=2 \pi F_{u}(r, \phi)=2 \pi\left(\alpha(r)-\alpha_{u}(\phi), r^{-1}\left(\beta(r)-\beta_{u}(\phi)\right) ;\right.
$$

cf. (5), (6), (7). The function $H_{u}$ is continuously differentiable on $D_{a}$, and there exists a $\rho>0$ such that

$$
\left|H_{u, x}(r, \phi, \epsilon)\right| \leq \rho, \quad u \in \Omega, \quad(r, \phi, \epsilon) \in D_{a} .
$$

Since $D_{a}$ is compact and convex, this implies that, for all $(\tilde{r}, \tilde{\phi}, \tilde{\epsilon}) \in D_{a}$ and $(r, \phi, \epsilon) \in D_{a}$, there holds

$$
\left|H_{u}(\tilde{r}, \tilde{\phi}, \tilde{\epsilon})-H_{u}(r, \phi, \epsilon)\right| \leq \rho|(\tilde{r}, \tilde{\phi}, \tilde{\epsilon})-(r, \phi, \epsilon)| .
$$

We are now ready to prove the following theorem.

Theorem 7.3. Under the conditions of Theorem 4.1, the maximum initial value of $2 \pi$-periodic solutions of (1) tends to $\bar{r}$ as $\epsilon \downarrow 0$, where $\bar{r}$ is defined by (11).

Proof. Since $(\bar{r}, 0)$ is a synchronization point of the bang-banger $\bar{u}$ defined by (13), there are $2 \pi$-periodic solutions of (1) with initial values $x(0)$ arbitrary close to $\bar{r}$ for small $\epsilon>0$. Especially, for every $\delta>0$, there are $2 \pi$-periodic solutions of (1) for the control $\bar{u}$ and sufficiently small $\epsilon>0$ with initial values $x(0)>\bar{r}-\delta$. It also follows that $m \geq \bar{r}$. Without loss of generality, we assume

$$
m>\bar{r}, \quad m>1 \text {. }
$$

It remains to be proved that, for every $\delta>0$ and $u \in \Omega$, there are no $2 \pi$-periodic solutions of (1) with an initial value $x(0) \geq \bar{r}+\delta$, for sufficiently small $\epsilon>0$. In view of (11) and (12), there exists a $\delta>0$ such that

$$
|\operatorname{sc}(r)|>|\operatorname{sc}(\bar{r}+\delta)|, \quad \text { for all } \bar{r}+\delta<r<m,
$$


and $\delta$ can be chosen arbitrarily small. We now prove that, for sufficiently small $\epsilon>0$ and all $u \in \Omega$, there are no $2 \pi$-periodic solutions of (4) with an initial value $r \geq \bar{r}+\delta$, by showing that, for all $u \in \Omega$,

$$
H_{u}(r, \phi, \epsilon) \neq 0 \text {, }
$$

on certain subregion of $D_{a}$. For all

$$
u \in \Omega, \quad r+\delta \leq r \leq m, \quad 0 \leq \phi \leq 2 \pi,
$$

we have

$$
\begin{aligned}
& \left|H_{u}(r, \phi, 0)\right| \\
& \quad=\mid\left(\alpha(r)-\alpha_{u}(\phi), r^{-1}\left(\beta(r)-\beta_{u}(\phi)\right) \mid\right. \\
& \quad \geq m^{-1}\left|(\alpha(r), \beta(r))-\left(\alpha_{u}(\phi), \beta_{u}(\phi)\right)\right|=m^{-1}\left|\operatorname{sc}(r)-A(\phi)\left(\alpha_{u}, \beta_{u}\right)\right| \\
& \quad=m^{-1}\left|A^{-1}(\phi) \operatorname{sc}(r)-\left(\alpha_{u}, \beta_{u}\right)\right| \geq m^{-1}(|\operatorname{sc}(\bar{r}+\delta)|-|\operatorname{sc}(\bar{r})|)=: \sigma(\delta)>0,
\end{aligned}
$$

where $A(\phi)$ is the matrix in (8), corresponding to a clockwise rotation over an angle $\phi$ around the origin. Let

$$
\epsilon_{\delta}:=\min \left(\epsilon_{a}, \epsilon_{m}, \rho^{-1} \sigma(\delta)\right),
$$

where $\rho$ is the upper bound for the functional matrix, as defined before. Now, if

$$
0<\epsilon<\epsilon_{\delta,} \quad u \in \Omega, \quad \bar{r}+\delta \leq r \leq m, \quad 0 \leq \phi \leq 2 \pi,
$$

then, from (28),

$$
\begin{aligned}
& \left|H_{u}(r, \phi, \epsilon)-H_{u}(r, \phi, 0)\right| \leq \rho|(r, \phi, \epsilon)-(r, \phi, 0)|=\rho \epsilon<\sigma(\delta), \\
& \left|H_{u}(r, \phi, 0)\right| \geq \sigma(\delta)
\end{aligned}
$$

consequently,

$$
\left|H_{u}(r, \phi, \epsilon)\right|>0 .
$$

In view of the definition of $m$ and $\epsilon_{m}$ and the $2 \pi$-periodicity of the right-hand side of (4) with respect to $\phi$, we may conclude that, if $0<\epsilon<\epsilon_{\delta}$, then, for all $u \in \Omega$, there are no $2 \pi$-periodic solutions of (4) with initial values

$$
r(0) \geq \bar{r}+\delta, \quad \phi(0) \in \mathbb{R},
$$

and hence no $2 \pi$-periodic solutions of (1) with initial values

$$
x(0) \geq \bar{r}+\delta .
$$

For the optimal controls, we have the following result. 
Theorem 7.4. Under the condition of Theorem 4.1, the optimal controls $\bar{u}_{\varepsilon} \in \Omega$, for which there exists a $2 \pi$-periodic solution with the maximum amplitude $A_{\epsilon}$ attained at $t=0$, satisfy

$$
\int_{0}^{2 \pi}\left|\bar{u}_{\epsilon}(t)-\bar{u}(t)\right| d t \rightarrow 0, \quad \epsilon \downarrow 0,
$$

where $\bar{u}$ is defined by (13).

Proof. For $0<\epsilon \leq \epsilon_{a}$, we have

$$
H_{\bar{u}_{\epsilon}}\left(A_{\epsilon}, 0, \epsilon\right)=0 \text {. }
$$

From this and (28), it follows that, for $\in \downarrow$,

$$
H_{\bar{u}_{\epsilon}}(\bar{r}, 0,0) \rightarrow 0 \text {; }
$$

hence,

$$
\begin{aligned}
& \alpha_{\bar{u}}-\alpha_{\bar{u}_{\varepsilon}}=\alpha(\bar{r})-\alpha_{\bar{u}} \rightarrow 0, \\
& \beta_{\bar{u}}-\beta_{\bar{u}_{\varepsilon}}=\beta(\bar{r})-\beta_{\bar{u}_{\varepsilon}} \rightarrow 0 .
\end{aligned}
$$

Let $s \in[0,2 \pi)$, such that

$$
\bar{u}(t)=\operatorname{sign}(\sin (t-s))
$$

then, using the fact that $\bar{u}$ and $\vec{u}_{\epsilon}$ are bang-bang controls, it follows that

$$
\begin{aligned}
& \int_{0}^{2 \pi}|\sin (t-s)|\left|\bar{u}_{\epsilon}(t)-\bar{u}(t)\right| d t \\
& \quad=\int_{0}^{2 \pi} \sin (t-s) \operatorname{sign}(\sin (t-s))\left|\bar{u}_{\epsilon}(t) \bar{u}(t)-1\right| d t \\
& \quad=\int_{0}^{2 \pi} \sin (t-s) \bar{u}(t)\left(1-\bar{u}_{\epsilon}(t) \bar{u}(t)\right) d t \\
& \quad=\int_{0}^{2 \pi} \sin (t-s)\left(\bar{u}(t)-\bar{u}_{\epsilon}(t)\right) d t \\
& =\cos s \int_{0}^{2 \pi} \sin t\left(\bar{u}(t)-\bar{u}_{\epsilon}(t)\right) d t \\
& -\sin s \int_{0}^{2 \pi} \cos t\left(\bar{u}(t)-\bar{u}_{\epsilon}(t)\right) d t \\
& =\left(\alpha_{\bar{u}}-\alpha_{\bar{u}_{\epsilon}}\right) \cos s-\left(\beta_{\bar{u}}-\beta_{\bar{u}_{\epsilon}}\right) \sin s \rightarrow 0, \quad \epsilon \downarrow 0 .
\end{aligned}
$$

Now, (29) follows from the fact that

$$
\left|\bar{u}_{\epsilon}(t)-\bar{u}(t)\right| \geq 0, \quad t \in[0,2 \pi] .
$$




\section{References}

1. Bollen, J. A. M., Maximum Amplitude of Controlled Oscillations, Twente University of Technology, Enschede, The Netherlands, Memorandum No. 338, 1981.

2. Roseau, M., Vibrations Nonlinéaires et Théorie de la Stabilité, Springer-Verlag, Berlin, Germany, 1966.

3. LeE, E. B., and MARkus, L., Foundations of Optimal Control Theory, John Wiley and Sons, New York, New York, 1967.

4. Boltyanskir, V. G., Mathematical Methods of Optimal Control, Holt, Rinehart, and Winston, New York, New York, 1971.

5. Coddington, E. A., and Levinson, N., Theory of Ordinary Differential Equations, McGraw-Hill, New York, New York, 1955.

6. Hautus, M. L. J., On the Uniform Boundedness of Solutions of Contingent Equations, Journal of Optimization Theory and Applications, Vol. 25, pp. 555$562,1978$.

7. BRIDGELAND, T. F., Contributions to the Theory of Generalized Differential Equations, I, Mathematical Systems Theory, Vol. 3, pp. 17-49, 1969.

8. Hautus, M. L. J., Necessary Conditions for Multiple Constraint Optimization Problems, SIAM Journal on Control, Vol. 11, pp. 653-669, 1973. 